\title{
Analysis of Tim-3 as a therapeutic target in prostate cancer
}

\author{
Yong-Rui Piao • Zhe-Hu Jin • Kui-Chang Yuan • \\ Xuan-Shun Jin
}

Received: 11 July 2014 / Accepted: 6 August 2014 / Published online: 15 August 2014

(C) The Author(s) 2014. This article is published with open access at Springerlink.com

\begin{abstract}
T cell immunoglobulin domain and mucin domaincontaining molecule 3 (Tim-3) is a newly discovered immunomodulatory, which plays an important role in immunity regulation. Recent evidence suggests that Tim-3 is differentially regulated in a variety of tumors and has a potential as a therapeutic target. The aim of this study was to investigate the effect of Tim-3 on the development of prostate cancer (PCa). Tim-3 expressing on peripheral CD4+ T and CD8 $+\mathrm{T}$ cells was analyzed by flow cytometry. The relationships between Tim-3 expression and clinicopathological features were analyzed. Immunohistochemical expression of Tim-3 was examined in our large numbers of paraffin-fixed prostate tissues. Flow cytometry revealed that expression of Tim-3 was significantly increased on both CD4+ and CD8 + T cells $1 \mathrm{~h}$ patients than that in benign prostate hyperplasia $(\mathrm{BPH})$ tients. Also, the level of Tim-3 on CD4+ T cells wa ssitively correlated with $\mathrm{CD} 8+\mathrm{T}$ cells in patients yrther a yses revealed that the levels of Tim-3 on $\mathrm{CD}^{2}+\mathrm{T}$ cells and $\mathrm{CD} 8+$ $\mathrm{T}$ cells exhibited different expression tterns ith terms of localization depending on patholeoical can of $\mathrm{PCa}$ and metastasis. Immunohistochemical in revealed that positive staining of Tim-3 in PC but litt or no staining of Tim-3 was observed in BPH e ithe am. T A-3 may affect the development and prores 1 or $\mathrm{Ca}$, which may provide knowledge for us Tim-3 a novel therapy for effective PCa manageme.t.

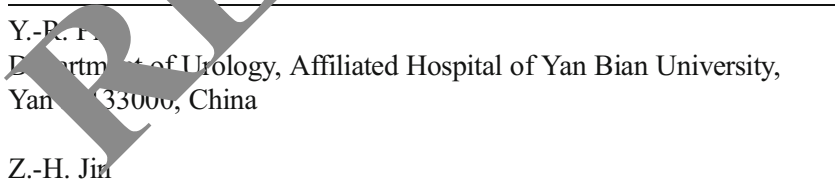

Department of Dermatology and Venereology, Affiliated Hospital of Yan Bian University, Yan Ji 133000, China

K.-C. Yuan · X.-S. Jin $(\bowtie)$

Department of Cardiology, Affiliated Hospital of Yan Bian University, No. 1327 Ju Zi Street, Yan Ji 133000, China

e-mail: yrpiao@126.com
\end{abstract}

Keywords Prostate cancer $\cdot T 3.4+$ i cells CD8+ T cells

Prostate cancer ( $\mathrm{PCo}$ ) is a he geneous disease with an estimated 241,740 new ases and 28,170 deaths related to this disease in 2012, 'mo - Ca the second most frequently diagnosed carcer and th ceond-leading cause of cancer death in men [1]. Ith o been a trend towards increased incidence and morbid $y$ of prostate cancer in Asia in the recent years [2]. $w^{3}$ the dev ropment of serum prostate-specific antigen (PSA) scre ng, MRI imaging, and new prostate biopsies protocols in cen years, the accuracy of detection and localization of p) sate tumors was improved obviously, but still $5 \%$ of cases present with metastatic lesions at the time of diagnosis [3]. The most common site of metastasis for $\mathrm{PCa}$ is the bone, and frequently, metastasis is symptomatic [4].

The main options for localized PCa are active surveillance, radical prostatectomy, and radiotherapy (RT) with or without adjuvant androgen deprivation therapy (ADT) [5]. Radical prostatectomy is the standard treatment for organ-confined tumors; however, even after seemingly complete removal of tumor, 20 to $30 \%$ of patients experience a recurrence, typically detected by a rise in PSA levels [6]. The incomplete understanding of molecular features of PCa might be one of the reasons for this unsatisfied situation, although recent gene expression studies have significantly improved our knowledge. Therefore, it is important to investigate the molecular mechanisms underlying the progression of $\mathrm{PCa}$ to provide effective strategies for the prevention and therapy of PCa.

T cell immunoglobulin and mucin-domain-containing molecule 3 (Tim-3), which could be identified as a specific cell surface marker of T-helper 1 (Th1) CD4+ T cells, is one of the Ig superfamily members and is preferentially expressed on fully differentiated Th1 lymphocytes but not on Th2 cells [7, 8]. Studies have shown that Tim-3 may not contribute to the T cell differentiation but might perform a critical function in the Th1 cell transportation $[9,10]$. Interaction between Tim-3 and its ligand galectin- 9 inhibits Th1 and Th17 responses and induces 
peripheral tolerance [11], suggesting an inhibitory role of Tim-3 in T cell responses. The soluble form of Tim- 3 would reduce the antigen-specific $\mathrm{T}$ cell responses and downregulate the antitumor immunity in vivo by inhibiting the Th1 responses.

Recent studies have shown an important role of Tim-3 T cell exhaustion in a variety of tumors. Tim-3 may play important roles in the development of non-small-cell lung cancer (NSCLC) [12]. It has been shown that Tim-3-expressing $\mathrm{CD} 4+$ and $\mathrm{CD} 8+\mathrm{T}$ cells are significantly increased in NSCLC patients. The expression levels of Tim-3 may be correlated with patients' survival [12]. Tim-3 and PD-1 are co-expressed on CD8 tumor-infiltrating lymphocytes (TILs) in mice bearing transplanted tumors as well as on NY-ESO-1specific CD8 $+\mathrm{T}$ cells in patients with advanced melanoma [13]. Blockade of the inhibitory Tim-3 pathway may prove useful in the treatment of a wide array of tumors, suggesting that Tim-3 pathway may act as one of the key factors in establishing T cell exhaustion [14]. However, there have been few studies reporting the expression of Tim-3 in PCa. This study was designed to explore the expression of Tim-3 in our large collection of clinical prostatic carcinoma samples and investigate its clinicopathological significance in PCa.

\section{Materials and methods}

\section{Patients and tissue specimens}

A total of 116 patients who had undergone radical rost tomy and bilateral lymphadenectomy at the $\mathrm{D}$ rtment Urology, Affiliated Hospital of Yan Bian Univ rsity, etween August 2001 and December 2010 and for whom a dival tissues were included in this study aime at detecting Tim-3 expression. No patient was managed pr arativ ly with either hormonal or radiation therapy and no secondary cancers were observed. Ninety-two cases or be ... prostate hyperplasia $(\mathrm{BPH})$ were obtained mer undergoing suprapubic prostatectomy or trans thre nlasnakinetic enucleation of prostate. The stages or can for all patients were determined by the Americar . nt Coml, attee on Cancer (AJCC) 2002 system. The specime vere examined by two staff pathologists who vere blinded 20 the clinical outcome and follow-up data. The atio of the specimen was performed according gut ines of the College of American Pathologists. D ffir ambedided tumor tissues and peripheral blood samples $\mathrm{m}$ these patients were evaluated. This study was approved sy the Ethics Committee of Affiliated Hospital of Yan Bian University. All patients provided informed consent.

Staining and flow cytometric analysis

Detection of Tim-3 was performed based on previously described methods. Peripheral blood sample was incubated for
$30 \mathrm{~min}$ in $4{ }^{\circ} \mathrm{C}$ in a dark room with monoclonal antibodies or isotype-matched control. Pe-Cy5-conjugated anti-human CD3, FITC-conjugated anti-human CD4, Pe-Cy5-conjugated anti-human $\mathrm{CD} 8$, phycoerythrin (PE)-conjugated rat IgG2a isotype control (Bioscience, San Diego, CA, USA), and monoclonal PE-conjugated anti-human Tim3 (R\&D Systems, Minneapolis, MN 55413, USA) were used for flow cytometric analysis. Cells were analyzed using a Beckman Coulter flow cytometer (FC500, Fullerton, CA, USA), and the data were analy usin the Cell Quest Program.

Immunohistochemistry

To quantify Tim-3 cells in large (nun rs of patients, paraffinembedded PCa samples were ocessur for immunohistochemistry. Specimens w re fixea $10 \%$ neutral buffered formalin, embedded i pa in, and cut into serial sections at a thickness of $3 \mu \mathrm{m}$-Daraffin- $y$ cedded tissues were dewaxed in xylene, rehy ratec by serial concentrations of ethanol, and then rinsed in $\mathrm{p}_{1}$ nllaw ouffer solution (PBS) followed by treatment with $3 \% \quad{ }_{2} \mathrm{O}_{2}$ to refrain endogenous peroxidase. After being . din a microwave at $750 \mathrm{~W}$ for $15 \mathrm{~min}$ to repair the til sue antigen, the sections were incubated with $\%$ norlyal goat serum at room temperature for 10 in to block non-specific reactions. This was loy ed by a PBS wash and incubation with primary ra monoclonal anti-human Tim-3 (clone 344823, 1/200, IgG2a, R\&D Systems) for $12 \mathrm{~h}$ at $4{ }^{\circ} \mathrm{C}$, and then with HRP-conjugated goat anti-rat $\operatorname{IgG}(1 / 500$, Invitrogen). After a PBS wash, the sections were developed in diaminobenzidine (DAB) substrate. The sections were then counter-stained in hematoxylin for $2 \mathrm{~min}$ and then dehydrated in ethanol and xylene before being mounted. Sections were re-prepared by EnVision immunohistochemical staining. PBS instead of primary antibodies was as negative control. Visualization was achieved with ABC-Elite Reagent (Sigma). The sections were counter-stained with Mayer's hematoxylin (Sigma). The nuclei were stained with $1 \%$ ammonium hydroxide. The numbers of Tim-3 cells were counted in five fields at $\times 400$ magnification.

\section{Statistical analysis}

Data analyses were performed using SPSS statistical package 15.0 (SPSS Inc, USA). Patient characteristics were expressed as the mean $\pm \mathrm{SD}$ for continuous variables, and as the count and percent for discrete variables. Data were analyzed using Student's $t$ test, Mann-Whitney non-parametric $U$ test, and standard chi-square analysis. The Pearson correlation analysis was used to calculate the correlation coefficient. A $P$ value less than 0.05 was considered significant. 


\section{Results}

Selected characteristics of the $116 \mathrm{PCa}$ patients and $92 \mathrm{BPH}$ controls are presented in Table 1. We investigated the expression of Tim-3 on CD4 $+\mathrm{T}$ cells and $\mathrm{CD} 8+\mathrm{T}$ cells from peripheral blood of 116 PCa patients and $92 \mathrm{BPH}$ controls. As shown in Fig. 1a, increased proportion of Tim-3+ cells was detected on CD4+ T cells in PCa patients than that in $\mathrm{BPH}$ patients (mean \pm SEM $4.02 \pm 0.46 \%$ vs $1.22 \pm 0.32 \%$, $P<0.001)$. Similarly, the expression of Tim- 3 on CD $8+$ T cells was also significantly elevated in $\mathrm{PCa}$ patients compared to BPH patients $(4.46 \pm 0.32 \%$ vs $0.82 \pm 0.20 \%, P<0.001)$ (Fig. 1b). We further investigated the correlation between Tim- 3 on CD4+ T cells and Tim- 3 on CD8+ T cells in $\mathrm{PCa}$ patients. Data revealed that the expression of Tim- 3 on CD4+ $\mathrm{T}$ cells was positively correlated with the level of Tim-3 on CD8+ T cells in our patient group $(r=0.646, P<0.001)$. These results suggest that Tim-3 maybe involved in the pathogenesis of PCa by its regulation on various immune cells.

We further investigated the levels of Tim-3 on CD4+ T cells and CD8+ T cells in the different groups of PCa patients. Data showed that the levels of Tim-3 on CD4+ T cells and $\mathrm{CD} 8+\mathrm{T}$ cells exhibited different expression patterns in terms of localization depending on pathological category of $\mathrm{PCa}$ and metastasis. We stratified localized PCa by the Gleason score into three subgroups, Gleason score $<7,=7$, and $>7$. In the localized PCa samples, levels of Tim-3 on CD4+ T cells and $\mathrm{CD} 8+\mathrm{T}$ cells appeared to be associated with a higher $\mathrm{G}^{1}$ ason score, which reached its predominance in Gleason $>7$. s (Fig. 2a). We further stratified localized PCa by ee subca egories on the basis of preoperative PSA level $<<1, \quad v / \mathrm{ml}$ as low risk, $10-20 \mathrm{ng} / \mathrm{ml}$ as intermediate ris/, and $>20 \mathrm{n} \varepsilon \mathrm{Al}$ as

\begin{tabular}{llll} 
Table 1 Correlations of Tim-3 expressi & & \\
features of $\mathrm{PCa}$ (the clinicopathological \\
\hline Variable
\end{tabular}

high risk. The analysis revealed that patients with the higher PSA level presented significantly higher Tim-3 expression on these cells, in which PCa patients with $>20 \mathrm{ng} / \mathrm{ml}$ PSA levels revealed significantly upregulated level of Tim-3 than the other stages (Fig. 2b). Next, we analyzed for Gleason score and preoperative PSA simultaneously. The analysis revealed that in the high-risk subcategory (PSA $>20 \mathrm{ng} / \mathrm{ml}$ ) higher levels of Tim-3 on CD4+ T cells and CD8+ T cells were associated with Gleason $>7$, and lower levels of Tim-3 on these cells were associated with Gleason $<7(P<0$.

In addition, the difference of levels of Timi- 3 on bot

$\mathrm{T}$ cells and CD8+ T cells between tissues types of metastasis (lymphnode, centarner ystem, or bone) also exists statistical signific ace $(P<0.0 /)$. No significant correlation, however, was foun hetwee/ levels of Tim-3 and age. Altogether, levels of $\mathrm{T} \quad 3$ orroun CD4+ T cells and $\mathrm{CD} 8+\mathrm{T}$ cells revealed ignifica elevation from PIN to localized and to meta atı $\mathrm{Ca}$ (Fig. 2c, d).

As levels of Tim 2 on both $74+\mathrm{T}$ cells and CD8+ T cells were basically etec $\mathrm{d}$ in peripheral blood of $\mathrm{PCa}$ and $\mathrm{BPH}$ patients, we ex tea vur studies further to include large numbers $\mathrm{f}$ paraffin ed prostate tissues with immunohistochemistry. In. examined i. $92 \mathrm{BPH}$ tissues and $116 \mathrm{PCa}$ tissues. We obed positi, e staining of Tim-3 in PCa; however, little or no stain g of Tim-3 was observed in BPH epithelium (Fig. 3). A tal f 82 of $116(70.69 \%)$ malignant cases showed positive sto, $\Delta$ ing for Tim-3, 18 of 92 (19.57 \%) benign cases showed positive, and the difference of Tim-3 expression between PCa and BPH was statistically significant $(P<0.001)$. There were higher numbers of Tim-3 cells in PCa tissues than BPH tissues. These results indicate that Tim-3 expression is increased on T cells infiltrating the PCa microenvironment.

A total of 116 archival PCa samples with intact clinicopathological data were identified for Tim-3 expression by immunohistochemistry and correlated with clinicopathological parameters. As a result, Tim-3 expression was positively correlated with the Gleason score and the concentration of PSA in blood serum and metastasis, while there was no significant relationship between Tim-3 level and variables such as age (Table 1).

\section{Discussion}

Tim-3 has emerged as a promising target for cancer immunotherapy [11]. Recent studies have focused on the role of Tim-3 expression in multiple pathological scenarios. Tim-3 is a molecule expressed on terminally differentiated Th1 cells but not on Th2 cells, which negatively regulate Th1 immunity [11]. It is also a phosphatidylserine receptor to mediate phagocytosis of apoptotic cells [15]. Recent studies showed that Tim-3 plays a significant role in tumor progression by maintaining the tumor immunosuppressive environment via regulatory $\mathrm{T}$ cells 
Fig. 1 Percentage of Tim-3 expression on $\mathrm{CD} 4+$ and $\mathrm{CD} 8+\mathrm{T}$ cells in $\mathrm{PCa}$ patients and $\mathrm{BPH}$ patients. a Percentage of Tim-3 expression on CD4+ T cells. b Percentage of Tim-3 expression on CD8+ T cells
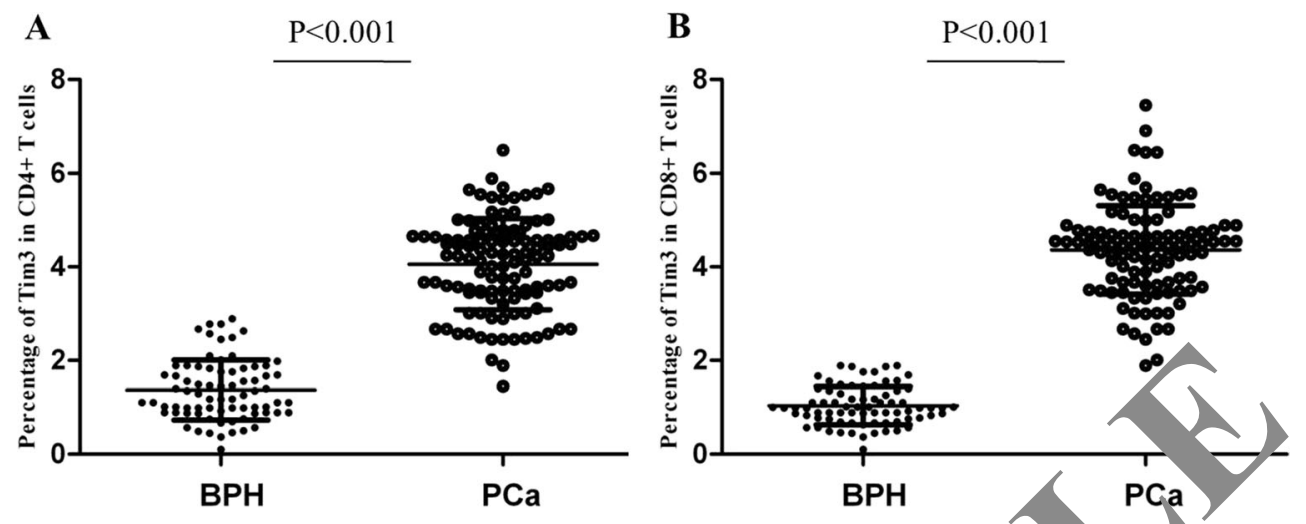

(T regs). Many studies have shown that dysregulation of Tim-3 expression on CD4+ T cells and CD8 + T cells is closely related to various tumors. For example, Wu et al. showed that Tim-3 expression on CD4+ T cells and CD8 + T cells was elevated in ovarian cancer [16]. Han et al. reported that the level of Tim-3 on CD4+ $\mathrm{T}$ cells was increased in glioma patients and was correlated with disease progression [17]. Recent studies have focused on the role of Tim-3 expression on $\mathrm{CD} 8+\mathrm{T}$ cells in peripheral blood as well as within tumors. Therefore, Tim-3 has emerged as a promising target for cancer immunotherapy. However, the nature of the Tim-3 signalis pathwy remains undefined in patients with PC In s stry, we evaluated the expression and clini 1 ro rance of the Tim-3 signaling pathway in a set o prostate samples, including BPH, PIN, ocah PCa, and metastatic PCa.

We first exanim Tim-3 xpression on various immune cells in PCa pa nts fiyther explored its correlation with disease activity. $h$ investigated the expression of Tim-3 on $\mathrm{CD} 4+\mathrm{T} d$ and $\mathrm{Cl} \delta+\mathrm{T}$ cells from peripheral blood of 116 PCa patients inc $2 \mathrm{BPH}$ controls. Our results show that the expression of Tim-3 on CD4+ T cells and CD8+ T cells was
Fig. 2 a Percentage of Tim-3 expression on $\mathrm{CD} 8+\mathrm{T}$ cells in the three subgroups of localized $\mathrm{PCa}$ samples, Gleason score $<7,=7$, and $>7$. b Percentage of Tim-3 expression on $\mathrm{CD} 8+\mathrm{T}$ cells in the in the three subgroups of localized PCa samples, PSA levels $<10 \mathrm{ng} / \mathrm{ml}, 10-20 \mathrm{ng} / \mathrm{ml}$, and $>$ $20 \mathrm{ng} / \mathrm{ml}$. c Percentage of Tim-3 expression on $\mathrm{CD} 4+\mathrm{T}$ cells in PIN, localized PCa, and metastatic PCa. d Percentage of Tim-3 expression on CD8* cells in PIN, localized PCa, anc metastatic $\mathrm{PCa}\left({ }^{*} P<\right.$ $* * P<0.01)$

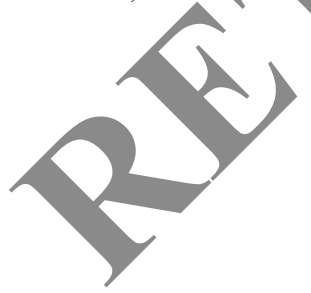

A

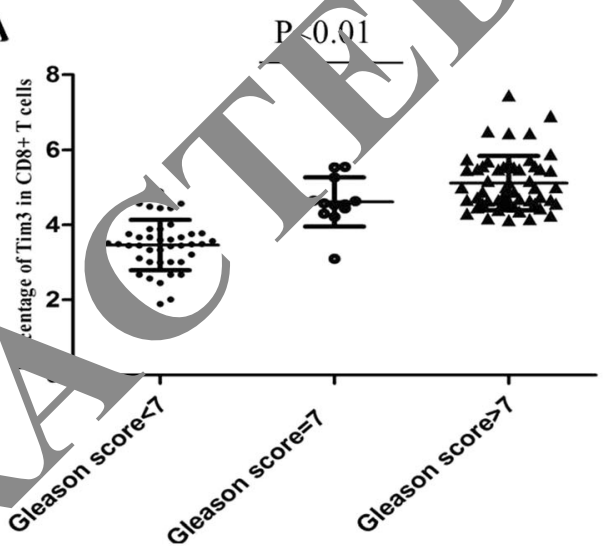

C

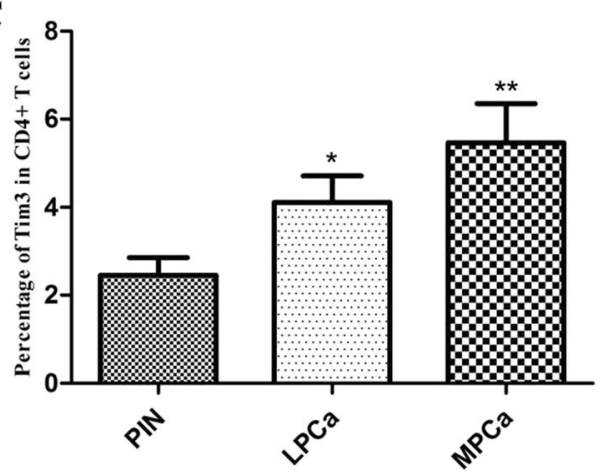

B

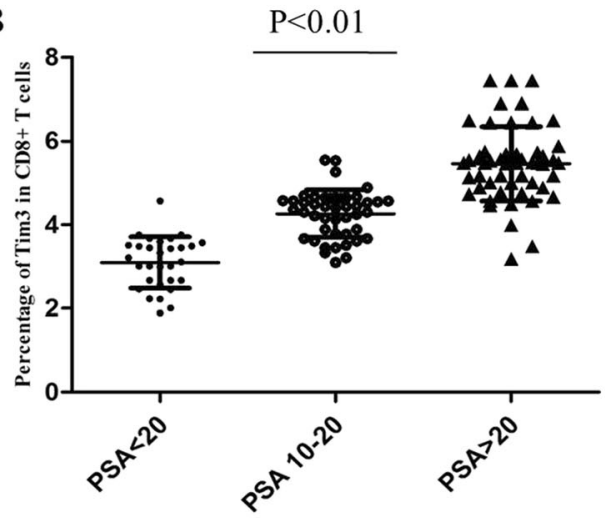

D

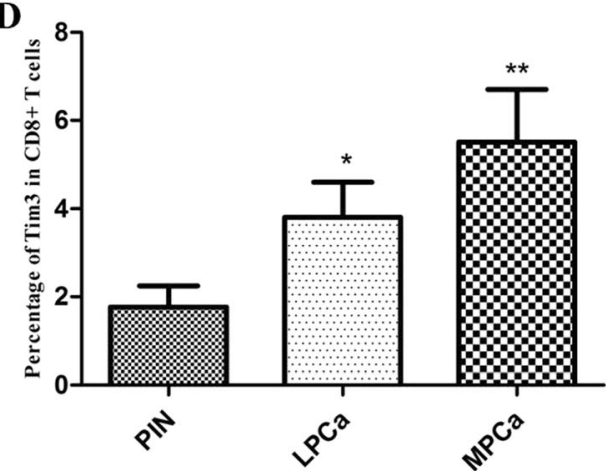


Fig. 3 Immunohistochemistry. a Positive staining of Tim-3 was observed in prostate cancer. $\mathbf{b}$ Little or no staining of Tim-3 was observed in the cytoplasm of benign prostate epithelium $($ Envision $\times 40)$
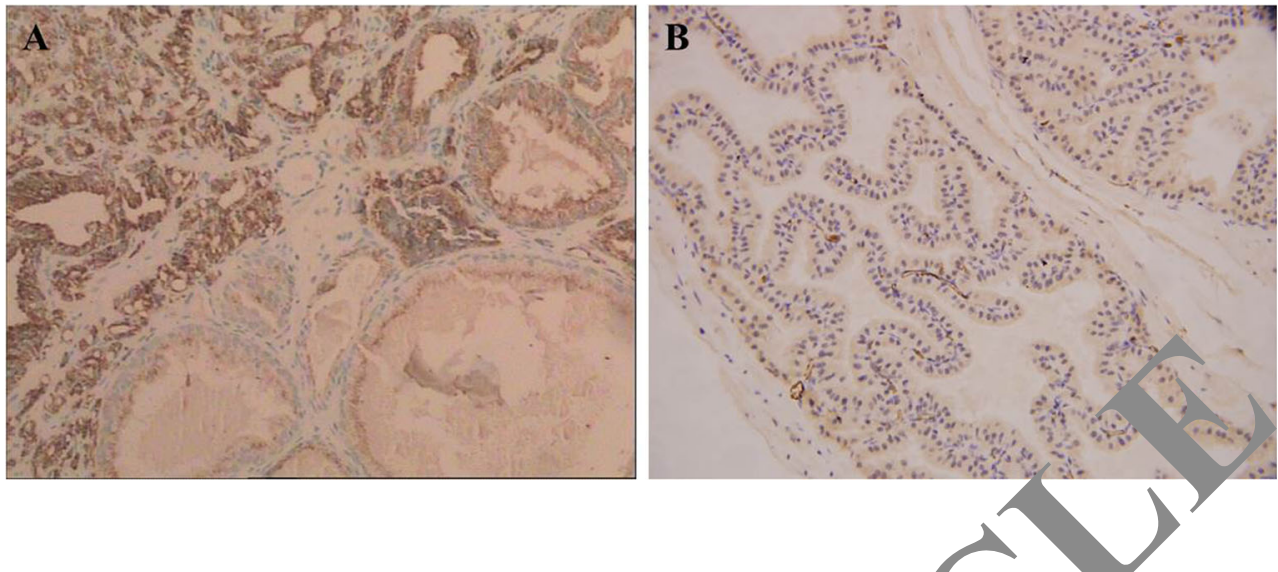

advanced disease stage, which pre licts a poor - r prognosis. However, further studies will be ded to understand the mechanism on how Tim-3 ma, ffec.... development and progression of $\mathrm{PCa}$, whic may p ide knowledge for using Tim-3 as a novel ther oy effective tumor management.

\section{Conflicts of interes ne}

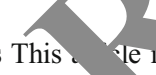

Open Access This le is ustributed under the terms of the Creative Commons Attribution whe which permits any use, distribution, and reproductio source are cr ditea.

interesting that we have found the levels of Tim-3 on CD4+ T cells and CD8 + T cells exhibited different expression patterns in terms of localization depending on pathological category of $\mathrm{PCa}$ and metastasis. In the localized PCa samples, levels of Tim-3 on CD4+ T cells and CD8+ T cells appeared to he associated with higher Gleason score and the higher pr operative PSA levels. In line with previous analysis, th lev ti Tim-3 on CD4+ T cells could be positively cor lated wh disease progression. In our PCa patients, leyes or ' n-3 on both CD4 $+\mathrm{T}$ cells and CD8 $+\mathrm{T}$ cells r veared sigh icant elevation from PIN to localized and to etastatic PCa, suggesting that Tim-3 may also act as an inc tor of the disease progression in PCa. In addition, 'he difference of levels of Tim-3 on both CD4+ T cells and CL ${ }^{7} 7 / 1 /$ is between tissues with or not with all types netast isis (lymphnode, central nervous system, or bo als existo statistical significance $(P<0.01)$.

Immunohistoc $\Lambda$ ical analy is revealed that positive staining of Tim-3 in PCa but lit r no staining of Tim-3 was observed in $\mathrm{BPH}$ epith ium. It is pos orble that Tim-3 expression is increased on T celis 1 ating the PCa microenvironment. Tim-3 expression posi 1. correlated with the Gleason score, the cono rati of PsA in blood serum, and the bone metastasis in $\mathrm{PC}$ a tients, while there was no significant relationship between Tim-3 vel and variables such as age.

In conclusion, our study identified an increased level of Tim-3 on both CD4+ and CD8+ T cells in peripheral blood as well as within tumors of PCa patients. Levels of Tim-3 on both CD4+ T cells and CD8 $+\mathrm{T}$ cells closely correlate with

\section{Refe nces}

1. Jemal A, Bray F, Center MM, Ferlay J, Ward E, Forman D. Global cancer statistics. CA Cancer J Clin. 2011;61:69-90.

2. Ren SC, Chen R, Sun YH. Prostate cancer research in China. Asian J Androl. 2013;15:350-3.

3. Saad F, Pantel K. The current role of circulating tumor cells in the diagnosis and management of bone metastases in advanced prostate cancer. Future Oncol. 2012;8:321-31.

4. Raheem O, Kulidjian AA, Wu C, Jeong YB, Yamaguchi T, Smith $\mathrm{KM}$, et al. A novel patient-derived intra-femoral xenograft model of bone metastatic prostate cancer that recapitulates mixed osteolytic and osteoblastic lesions. J Transl Med. 2011;9:185.

5. Heidenreich A, Bellmunt J, Bolla M, Joniau S, Mason M, et al. EAU guidelines on prostate cancer. Part 1: screening, diagnosis, and treatment of clinically localized disease. Eur Urol. 2011;59:61-71.

6. Pontes-Junior J, Reis ST, de Oliveira LC, Sant'anna AC, Dall'oglio MF, Antunes AA, et al. Association between integrin expression and prognosis in localized prostate cancer. Prostate. 2010;70:1189-95.

7. Anderson AC. Tim-3, a negative regulator of anti-tumor immunity. Curr Opin Immunol. 2012;24:213-6.

8. Anderson AC, Anderson DE, Bregoli L, Hastings WD, Kassam N, Lei C, et al. Promotion of tissue inflammation by the immune receptor Tim-3 expressed on innate immune cells. Science. 2007;318:1141-3.

9. Kikushige Y, Akashi K. TIM-3 as a therapeutic target for malignant stem cells in acute myelogenous leukemia. Ann N Y Acad Sci. 2012;1266:118-23.

10. Kozlowska A, Mackiewicz J, Mackiewicz A. Therapeutic gene modified cell based cancer vaccines. Gene. 2013;525:200-7. 
11. Sakuishi K, Jayaraman P, Behar SM, Anderson AC, Kuchroo VK. Emerging Tim-3 functions in antimicrobial and tumor immunity. Trends Immunol. 2011;32:345-9.

12. Gao X, Zhu Y, Li G, Huang H, Zhang G, Wang F, et al. TIM-3 expression characterizes regulatory $\mathrm{T}$ cells in tumor tissues and is associated with lung cancer progression. PLoS One. 2012;7(2):e30676.

13. Fourcade J, Sun Z, Benallaoua M, Guillaume P, Luescher IF, Sander C, et al. Upregulation of Tim-3 and PD-1 expression is associated with tumor antigen specific CD8 $+\mathrm{T}$ cell dysfunction in melanoma patients. J Exp Med. 2010;207:2175-86.

14. Jones RB, Ndhlovu LC, Barbour JD, Sheth PM, Jha AR, Long BR, et al. Tim-3 expression defines a novel population of dysfunctional T cells with highly elevated frequencies in progressive HIV-1 infection. J Exp Med. 2008;205:2763-79.

15. Zhu C, Anderson AC, Schubart A, Xiong H, Imitola J, Khoury SJ, et al. The Tim-3 ligand galectin-9 negatively regulates $\mathrm{T}$ helper type 1 immunity. Nat Immunol. 2005;6: $1245-52$.

16. Wu J, Liu C, Qian S, Hou H. The expression of Tim-3 in peripheral blood of ovarian cancer. DNA Cell Biol. 2013;32: 648-53.

17. Han S, Feng S, Xu L, Shi W, Wang X, Wang H, et al. Tim-3 on peripheral CD4 $(+)$ and $\mathrm{CD} 8(+) \mathrm{T}$ cells is involved in the evelopment of glioma. DNA Cell Biol. 2014;33:245-50.
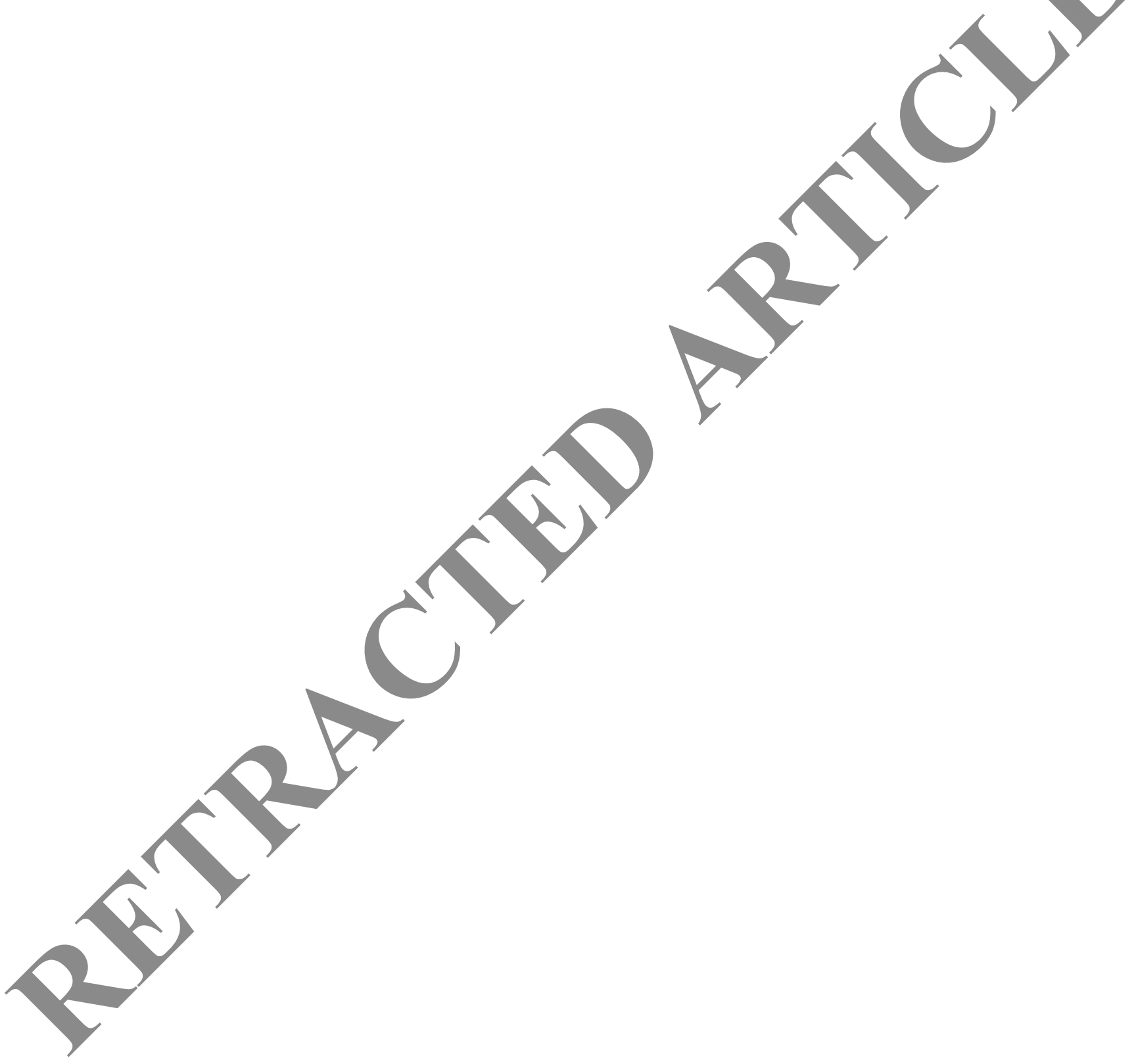